\title{
Coating Evaporated MAPI Thin Films with Organic Molecules: Improved Stability at High Temperature and Implementation in High- ¿Efficiency Solar Cells
}

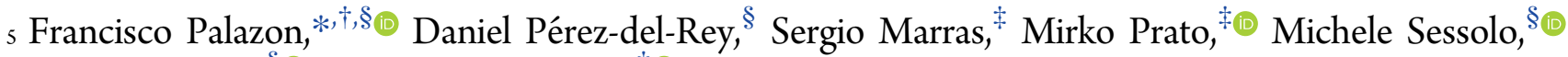 \\ ${ }_{6}$ Henk J. Bolink, ${ }^{\S}$ and Liberato Manna ${ }^{\dagger}$ \\ $7{ }^{\dagger}$ Nanochemistry Department, and ${ }^{\ddagger}$ Materials Characterization Facility, Istituto Italiano di Tecnologia, Via Morego 30, 16163 Genova, \\ 8 Italy \\ $9{ }^{\S}$ Instituto de Ciencia Molecular, ICMol, Universidad de Valencia, C/Catedrático J. Beltrán 2, 46980 Paterna, Spain
}

11 ABSTRACT: Methylammonium lead iodide (MAPI) has proven to

12 be an exceptional light-absorber for single-junction thin-film solar 13 cells. Nonetheless, degradation induced by environmental agents (air, 14 moisture, heat) limits the stability of this hybrid perovskite. Here, we 15 demonstrate that coating evaporated MAPI thin films with different 16 hydrophobic molecules leads to a significant improvement in their 17 stability. We especially investigated the degradation of MAPI and the 18 subsequent formation of $\mathrm{PbI}_{2}$ at $150{ }^{\circ} \mathrm{C}$ by in situ $\mathrm{XRD}$ analysis and 19 showed that this transformation is remarkably slowed down in films 20 coated with trioctyl phosphine oxide and tridodecyl methylammonium iodide. This enhances the processability of such films, which is an important aspect for the fabrication of thin-film devices. Eventually, we demonstrate that such protected films can be implemented in single-junction $n-i-p$ solar cells without any loss in the device efficiency.

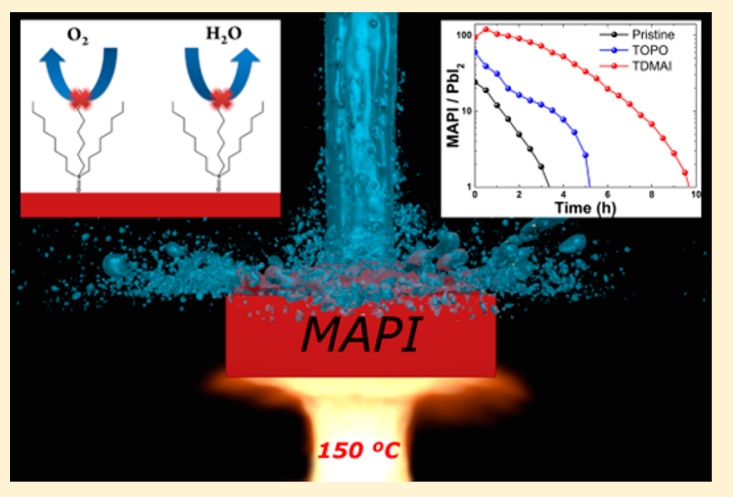

"A $\mathrm{s}$ widely reported in the past few years, methylammonium lead iodide $\left(\mathrm{CH}_{3} \mathrm{NH}_{3} \mathrm{PbI}_{3}\right.$; MAPI) is an excellent material for single-junction solar cells and other 29 optoelectronic devices. ${ }^{1-4}$ The outstanding performances of 30 these devices can be linked to several photophysical properties 31 of perovskites, such as their high absorption coefficient, narrow 32 bandgap, and high charge diffusion lengths, ${ }^{5,6}$ together with the 33 ease of fabrication and low cost of precursors. ${ }^{7}$ Nonetheless, the 34 widespread use of MAPI is currently severely hindered by its 35 well-known instability. Indeed, oxygen, water (moisture), and 36 heat among other factors are known to lead to a fast 37 degradation of MAPI thin films. ${ }^{8,9}$ In order to mitigate this 38 instability, one possible approach is to alter the material 39 composition, as already demonstrated in many reports. These 40 modifications include the partial substitution of $\mathrm{I}^{-}$anions with $41 \mathrm{Br}^{-}$anions, ${ }^{10}$ the partial or total substitution of MA cations 42 with other organic or inorganic monovalent cations such as 43 formamidinium $\left(\mathrm{CH}\left(\mathrm{NH}_{2}\right)_{2}^{+} ; \mathrm{FA}\right)$, cesium $\left(\mathrm{Cs}^{+}\right)$, or rubidium $44\left(\mathrm{Rb}^{+}\right),{ }^{11-13}$ or the incorporation of $2 \mathrm{D}$ perovskites. ${ }^{14-18}$ These 45 substitutions can lead to rather complex formulations 46 comprising up to seven different ions whose relative amounts need to be well controlled. ${ }^{19}$ Furthermore, bromide-based and 47 $2 \mathrm{D}$ perovskites have a higher bandgap than their $3 \mathrm{D}$ iodide 48 counterparts, which is not beneficial for photovoltaic (PV) 49 applications. ${ }^{14-18}$ Hence, it is critical to explore new ways of 50 enhancing the stability of MAPI films without necessarily 51 altering their overall composition. Because degradation often 52 initiates at the surface of the films through its exposure to 53 external species (e.g., moisture), ${ }^{20}$ one possible strategy is to 54 chemically modify the perovskite surface with different organic 55 molecules. Yang et al. ${ }^{21}$ showed that coating the surface of 56 solution-processed MAPI thin films with different hydrophobic 57 molecules leads to an increased stability in a high-moisture 58 environment at room temperature. The same approach has 59 recently been developed by Wang et al., ${ }^{22}$ while deQuilettes et 60 $\mathrm{al}^{23}$ found that surface modification with Lewis base molecules 61 leads to efficient passivation of the nonradiative surface trap 62 states. Huang et al. also noticed an increase of initial efficiency 63

Received: February 5, 2018

Accepted: March 9, 2018

Published: March 9, 2018 
64 as well as stability in single-junction solar cells with a similar 65 method. $^{24} \mathrm{~A}$ critical aspect to consider for the widespread 66 implementation of MAPI in thin-film devices is its stability at 67 high temperature $\left(>100{ }^{\circ} \mathrm{C}\right)$. Although this may seem 68 irrelevant for typical operation conditions, it is important to 69 evaluate the stability at high temperature in view of the 70 fabrication of optoelectronic devices. Indeed, the typical 71 fabrication processes of thin-film devices require the deposition 72 of several additional layers on top of the active (perovskite) film 73 such as charge extracting layers, electrodes, or encapsulation 74 materials. Depending on the process and the material, these 75 further depositions may require temperatures well above 100 $76{ }^{\circ} \mathrm{C}$. In this Letter, we have coated vacuum-deposited MAPI thin 77 films with trioctyl phosphine oxide (TOPO) and tridodecyl 78 methylammonium iodide (TDMAI), adapting reported proto79 cols by deQuilletes et al. ${ }^{23}$ and Yang et al., ${ }^{21}$ respectively. We 80 demonstrate in both cases the presence of ultrathin layers of 81 TOPO and TDMAI through direct chemical characterization 82 by X-ray photoelectron spectroscopy (XPS). Then, we show by 83 in situ X-ray diffraction (XRD) that the so-coated films have a 84 drastically enhanced stability in air at $150{ }^{\circ} \mathrm{C}$. Eventually, we 85 demonstrate that the presence of these layers is not 86 incompatible with the good operation of PV devices. As a 87 proof-of-principle, we fabricate single-junction solar cells with 88 pristine and coated MAPI in $\mathrm{n}-\mathrm{i}-\mathrm{p}$ configurations that show 89 promising efficiency and stability. ${ }^{25}$

90 MAPI thin films were prepared by dual-source vacuum 91 deposition on glass substrates, as explained in the experimental 92 section. Then, the films were coated either with TDMAI or 93 TOPO. Adapting reported protocols, ${ }^{21,23}$ TDMAI-coated 94 samples were realized by fully immersing the MAPI thin films 95 in a $25 \mathrm{mM}$ solution in isopropanol, while the TOPO-coated 96 samples were prepared by a single spin-coating step of a $25 \mathrm{mM}$ 97 TOPO solution in toluene (see the Supporting Information for 98 more details).

99 Figure 1a shows wide XPS spectra of pristine and TDMAI100 coated films. Although TDMAI does not have any distinctive
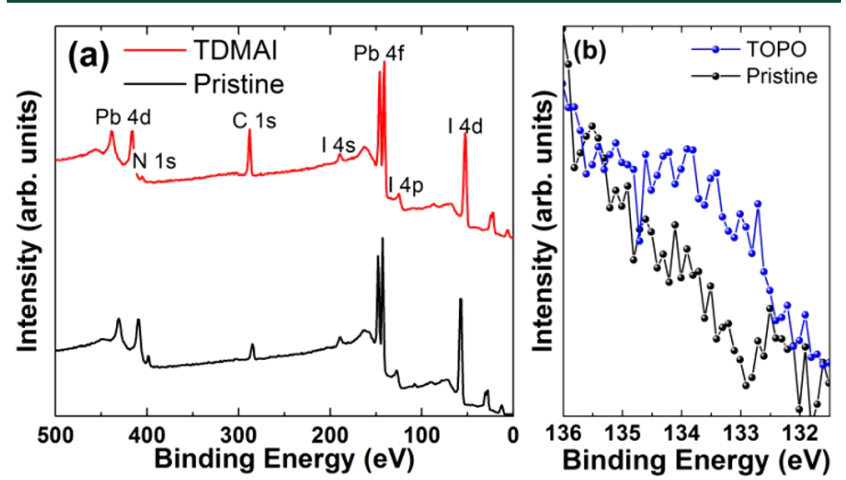

Figure 1. XPS characterization of pristine MAPI films as well as films coated with TDMAI and TOPO. (a) Wide scans of pristine and TDMAI samples and (b) narrow scans of the $P 2 p$ region of pristine and TOPO samples (see Figure S1 for XPS characterization of a sample with a thicker layer of TOPO).

101 chemical element other than the ones present already in the 102 MAPI film, XPS reveals an increase in the carbon content of the 103 film surface after functionalization (the $\mathrm{C}: \mathrm{Pb}$ ratio increases by 104 a factor of $>3$ ), which proves the attachment of TDMAI 105 molecules to the surface. Figure $1 \mathrm{~b}$ shows XPS narrow scans of 106 the region corresponding to $2 \mathrm{p}$ orbitals from phosphorus ( $\mathrm{P}$
$2 p)$. The increase in signal is evident proof of the presence of 107 TOPO (see Figure S1 for XPS characterization of a film coated 108 with a $50 \mathrm{mM}$ solution of TOPO). Yet, the signal remains very 109 low, which is expected for a thin layer. This is important in 110 order to allow charge tunneling with electron or hole transport 111 layers in solar cells and, generally speaking, to allow the use of 112 such coated films in optoelectronic devices. Next, the stability 113 of pristine and coated films at $150{ }^{\circ} \mathrm{C}$ was evaluated by in situ 114 XRD measurements (Figure 2). As mentioned before, thermal $115 \mathrm{f} 2$

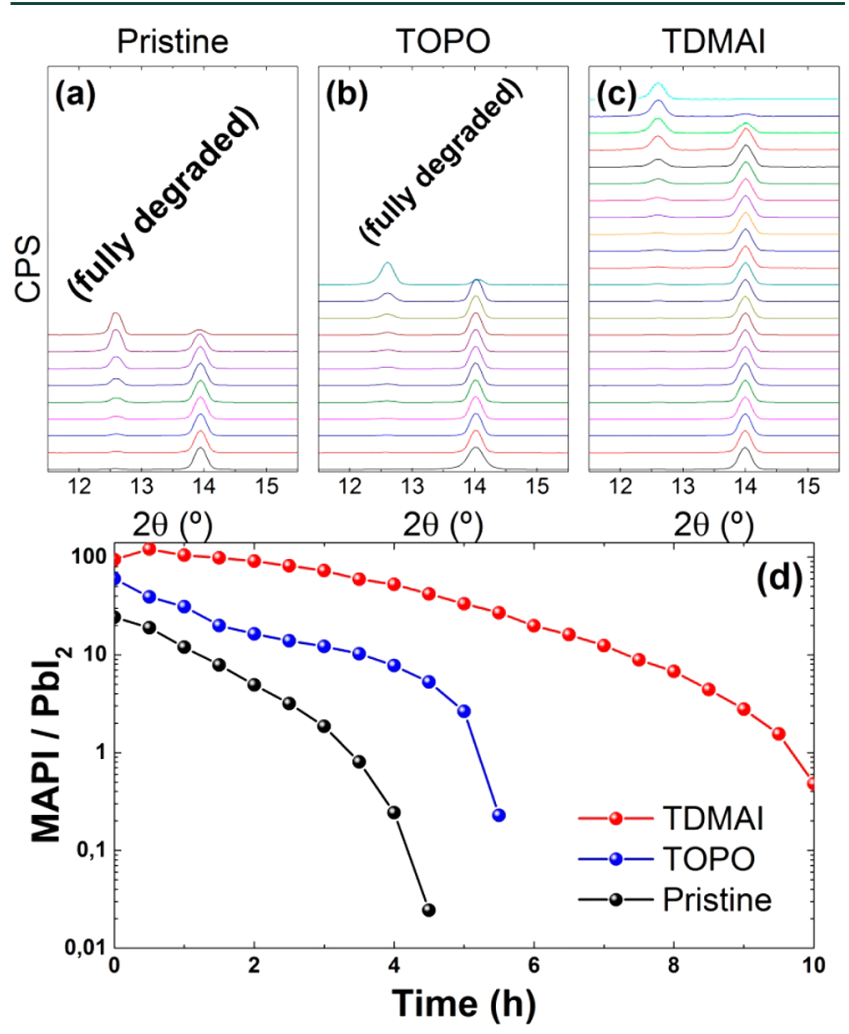

Figure 2. XRD characterization of sample degradation at $150{ }^{\circ} \mathrm{C}$. $(a-d)$ Diffractograms of pristine (a) and coated (b,c) films over time. Initial scan at the bottom, $30 \mathrm{~min}$ between each scan. (d) Intensity ratio between the main perovskite and $\mathrm{PbI}_{2}$ peaks $(2 \theta=$ 14.0 and $12.6^{\circ}$, respectively), computed from panels $(a-c)$.

annealing above $100{ }^{\circ} \mathrm{C}$ is a common step in many thin-film 116 fabrication procedures (atomic layer deposition, sputtering, 117 etc.); hence, it is technologically relevant to evaluate the 118 stability of the thin films at such a temperature.

Figure $2 \mathrm{a}-\mathrm{c}$ presents the XRD patterns over time of pristine 120 and treated films at a fixed temperature of $150{ }^{\circ} \mathrm{C}$, in the range 121 $2 \theta=12-15^{\circ}$. This allows one to evidence the loss of MAPI 122 (vanishing of the peak at $\left.2 \theta=14.0^{\circ}\right)^{26}$ and the subsequent rise 123 of $\mathrm{PbI}_{2}$ (rise of the peak at $2 \theta=12.6^{\circ}$ ). In order to have 124 semiquantitative analysis of the degradation, we computed the 125 intensity ratio of MAPI and $\mathrm{PbI}_{2}$ main peaks $(2 \theta=14.0$ and 126 $12.6^{\circ}$ ), which is proportional to the relative amount of both 127 species in the sample. This ratio is plotted in Figure 2d. The 128 initial MAPI/ $\mathrm{PbI}_{2}$ ratio is not infinite because from the very 129 first scan a slight contribution from $\mathrm{PbI}_{2}$ is already observed. 130 This is very common in perovskite films and can be due either 131 to impurities during the synthesis/deposition or even to a slight 132 degradation already occurring during exposure of the samples 133 to air prior to the first analysis. In any case, the initial fraction of 134 $\mathrm{PbI}_{2}$ is low, as can be seen from Figure 2. This fraction 135 
136 increases over time for all samples, though not at the same 137 pace. Indeed, treated samples are significantly more stable.

138 As an arbitrary measure, we may consider the time $t_{10}$ by 139 which the $\mathrm{PbI}_{2}$ peak has reached an intensity equal to $10 \%$ of 140 the main perovskite peak. As can be seen in Figure $2 \mathrm{~d}, t_{10}$ goes 141 from less than $2 \mathrm{~h}$ for pristine MAPI to $4 \mathrm{~h}$ for TOPO-treated 142 films and to $8 \mathrm{~h}$ for TDMAI-treated ones. This reveals that 143 coated MAPI films can withstand high temperature much better 144 than pristine ones. In other terms, surface coating with organic 145 molecules allows the film to be processed at temperatures 146 above $100{ }^{\circ} \mathrm{C}$ for longer times, during which the deposition of 147 further materials or hot encapsulation of devices can take place. 148 This stability test at high temperature can also be seen as an 149 accelerated test of the degradation that occurs already at room 150 temperature. Indeed, at room temperature, pristine films were 151 found to be quickly degraded $\left(t_{10}<2\right.$ weeks), while coated 152 ones were not significantly altered even after 3 weeks (see 153 Figure S2). These results suggest a combined contribution from 154 the coating molecule's head group and alkyl chains to the 155 enhanced stability of the film. Indeed, as previously suggested 156 by others, ${ }^{21}$ the hydrophobic nature of the alkyl chains present 157 on both TDMAI and TOPO may block moisture-derived 158 degradation. However, the fact that TDMAI-coated samples are 159 more stable than TOPO-coated ones suggests that the higher 160 affinity of the MAI head group (which can perfectly fit in the 161 MAPI structure) in contrast with the $\mathrm{P}=\mathrm{O}$ group of TOPO 162 (which is not known to specifically bind to MAPI) may also 163 play an important role. More detailed investigations on these 164 fundamental aspects are however needed to draw unambiguous 165 conclusions.

166 We fabricated different solar cells with pristine and treated 167 MAPI. Although TDMAI yielded the highest material stability 168 (Figure 2), we were not able to fabricate efficient and 169 reproducible devices with it. Two reasons may explain this 170 evidence: either the TDMAI layer was too thick (which would 171 also explain the highest stability) and did not allow charge 172 transfer in the device or the process induced other negative 173 consequences in the film such as the occurrence of pinholes 174 that lead to short circuits. The first hypothesis is not in 175 accordance with our XPS analysis, which reveals a strong signal 176 for lead (Figure 1a) and suggests that the MAPI film is not 177 covered by a thick $(>5 \mathrm{~nm})$ uniform insulating layer. On the 178 other hand, because the coating process, adapted from Yang et 179 al., $^{21}$ is based on full immersion of the film in a fairly polar 180 solvent (isopropanol), which might partially degrade the 181 perovskite, the second hypothesis seems more plausible.

182 In contrast, we were able to fabricate solar cells with TOPO183 coated MAPI films. Indeed, although TOPO is insulating, 184 charge extraction by tunneling through an insulating layer can 185 be achieved if the layer is thin enough, as also observed by 186 others. $^{24,27}$ The TOPO layer thickness can be estimated by the 187 intensity drop of I $3 \mathrm{~d}$ and $\mathrm{Pb} 4 \mathrm{f}$ XPS peaks (see Figure S3). 188 Indeed, as MAPI is covered by an organic layer, photoelectrons 189 from the underlying lead and iodine atoms will be absorbed 190 following Beer-Lambert law. Hence, the intensity of the peaks 191 (or rather peak areas) will decrease as follows: $A_{\mathrm{TOPO}}(\mathrm{X})=$ $192 A_{\text {Pristine }}(\mathrm{X}) \mathrm{e}^{-\lambda / d}$, where $A_{\text {TOPO }}(\mathrm{X})$ and $A_{\text {Pristine }}(\mathrm{X})$ are the area 193 corresponding to XPS peak X (in this case I $3 \mathrm{~d}$ and $\mathrm{Pb} 4 \mathrm{f}$ ) in 194 the presence or absence of TOPO, $\lambda$ is the inelastic mean free 195 path of photoelectrons in the TOPO layer, and $d$ is the 196 thickness of the layer. If we assume $\lambda=3 \mathrm{~nm}$ as a common 197 value for organic material, ${ }^{28}$ then we obtain $d=\lambda \times \ln \left(A_{\text {Pristine }}\right.$ (I $\left.1983 \mathrm{~d}) / A_{\mathrm{TOPO}}(\mathrm{I} 3 \mathrm{~d})\right)=0.5 \mathrm{~nm}$ (considering iodine peaks) or $d=\lambda$ $\times \ln \left(A_{\text {Pristine }}(\mathrm{Pb} 4 \mathrm{f}) / A_{\text {Tоро }}(\mathrm{Pb} 4 \mathrm{f})\right)=0.6 \mathrm{~nm}$ (considering lead 199 peaks). Both values are close to and consistent with a single 200 monolayer of TOPO. As we will show hereafter, this monolayer 201 of TOPO does not degrade the device performance (Figure 3). $202 \mathrm{f} 3$
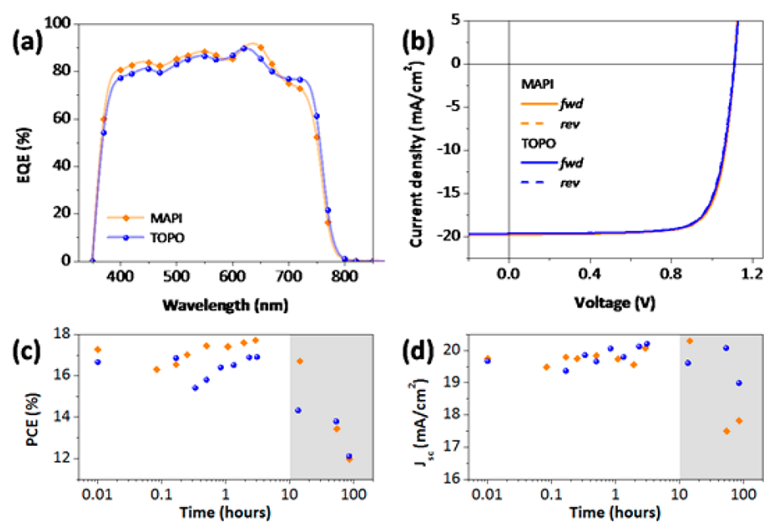

Figure 3. (a) EQE spectra and (b) $J-V$ curves under illumination for solar cells with pristine MAPI (orange squares) and MAPI functionalized with TOPO (blue spheres). (c) Efficiency and (d) photocurrent of the same cells recorded over time during air exposure and without any encapsulation. See Figure S5 for more statistics on TOPO samples.

In contrast, when a more concentrated solution of TOPO is 203 used, resulting in a slightly thicker layer (see Figure S1), the 204 device performs significantly worse (see Figure S4). This 205 highlights the critical importance of the coating layer thickness. 206 The cells were prepared with vacuum-deposited MAPI in the 207 $\mathrm{n}-\mathrm{i}-\mathrm{p}$ configuration, avoiding the use of doped transport layers 208 in order to be able to test their stability in air without 209 encapsulation. For this purpose, we coated the indium tin oxide 210 (ITO)/glass substrates with a bilayer of $\mathrm{TiO}_{2}$ nanoparticles (50 211 $\mathrm{nm})$ in combination with a thin $(10 \mathrm{~nm})$ C60 film in contact 212 with MAPI. The fullerene layer was introduced in order to 213 reduce carrier recombination at the $\mathrm{TiO}_{2}$ electron transport 214 layer. ${ }^{13}$ After deposition of the perovskite, a double hole 215 transport layer composed of $N 4, N 4, N 4^{\prime \prime}, N 4^{\prime \prime}$-tetra $\left(\left[1,1^{\prime}-216\right.\right.$

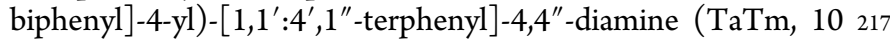
$\mathrm{nm})$ and $\mathrm{MoO}_{3}(10 \mathrm{~nm})$ was sublimated onto the MAPI, and 218 the device was finished with a gold electrode $(100 \mathrm{~nm})$. Figure 219 $3 a$ shows the external quantum efficiency (EQE) spectra for 220 solar cells with and without the TOPO layer. Their spectral 221 response was fairly similar (small differences are due to batch- 222 to-batch variations) and above $80 \%$ over all of the visible 223 spectrum. This resulted in a short-circuit current density of 19.7224 $\mathrm{mA} / \mathrm{cm}^{2}$ for both devices. The current density versus voltage 225 $(J-V)$ curves recorded under 1 sun illumination (Figure 3b) 226 were practically identical in the presence of TOPO, suggesting 227 that charge transport and recombination are substantially 228 unaltered by its presence. Also, the $J-V$ curves recorded in 229 forward (fwd) and reverse (rev) are virtually hysteresis-free. 230 The open-circuit voltage $\left(V_{\text {oc }}\right)$ was as high as $1100 \mathrm{mV}$, and we 231 noted only a small reduction in the fill factor (from 78.8 to 232 $77.1 \%$ ) upon TOPO treatment. The resulting power 233 conversion efficiency (PCE) was $17.2 \%$ for the solar cell 234 using pristine MAPI and $16.7 \%$ for the one with TOPO- 235 modified perovskite.

236

We tested the stability of the cells in air without any 237 encapsulation, measuring the $J-V$ curves over time. We 238 observed a first drop in the efficiency followed by a slow 239 
240 recovery during the first $10 \mathrm{~h}$ (Figure 3c), after which the 241 device performance started to drop. While the PCE of the 242 devices decreased in a similar manner independently of the use 243 of the TOPO layer, we observed how the cells with TOPO244 modified MAPI retained the photocurrent for a much longer 245 time (Figure 3d), with a slower decrease as compared to the 246 pristine MAPI cells. As the photocurrent is mainly determined 247 by the rate of carrier generation within the perovskite, this 248 might indicate that the presence of TOPO can alleviate the 249 perovskite degradation when incorporated in a working device, 250 in analogy with the results presented for simple perovskite films 251 (Figure 2).

252 In summary, we have shown that surface coating with organic 253 molecules can greatly enhance the stability of pure MAPI thin 254 films at high temperature, as well as room temperature. This 255 methodology is promising for enhancing both the processability 256 window of the material and the long-term operational stability. 257 Indeed, we have shown that efficient solar cells could be 258 fabricated with functionalized MAPI thin films. While the 259 photocurrent was observed to be maintained for a longer time 260 during air exposure of the cells, further developments, 261 optimizing the choice of organic molecules and the deposition 262 method, should allow one to enhance the stability while 263 minimizing efficiency losses. Furthermore, a deeper study on 264 halide perovskite surface functionalization would certainly shed 265 more light on the binding mechanisms of different molecules 266 on perovskites.

\section{ASSOCIATED CONTENT}

\section{S Supporting Information}

269 The Supporting Information is available free of charge on the 270 ACS Publications website at DOI: 10.1021/acsenergy271 lett.8b00193.

272 Experimental details, XPS of samples with different 273 amounts of TOPO, XRD of samples aged at room 274 temperature, and $J-V$ curves of devices based on 275 different amounts of TOPO (PDF)

\section{$276 \square$ AUTHOR INFORMATION}

277 Corresponding Author

278 *E-mail: Francisco.palazon@uv.es.

279 ORCID

280 Francisco Palazon: 0000-0002-1503-5965

281 Mirko Prato: 0000-0002-2188-8059

282 Michele Sessolo: 0000-0002-9189-3005

283 Henk J. Bolink: 0000-0001-9784-6253

284 Liberato Manna: 0000-0003-4386-7985

285 Notes

286 The authors declare no competing financial interest.

\section{ACKNOWLEDGMENTS}

288 The research leading to these results received funding from the 289 seventh European Community Framework Programme under 290 Grant Agreement No. 614897 (ERC Consolidator Grant 291 "TRANS-NANO"), the framework Programme for Research 292 and Innovation Horizon 2020 (2014-2020) under the Marie 293 Skłodowska-Curie Grant Agreement PerovSAMs No. 747599, 294 and project INFORM (Grant 675867). Financial support is 295 also acknowledged from the Spanish Ministry of Economy and 296 Competitiveness (MINECO) via the Unidad de Excelencia 297 María de Maeztu MDM-2015-0538 and MAT2014-55200,
PCIN-2015-255, and the Generalitat Valenciana (Prometeo/ 298 2012/053). M.S. thanks the MINECO for his RyC contract. 299

\section{REFERENCES}

300

(1) Stranks, S. D.; Snaith, H. J. Metal-Halide Perovskites for 301 Photovoltaic and Light-Emitting Devices. Nat. Nanotechnol. 2015, 10302 (5), 391-402.

(2) Zhao, Y.; Zhu, K. Organic-inorganic Hybrid Lead Halide 304 Perovskites for Optoelectronic and Electronic Applications. Chem. Soc. 305 Rev. 2016, 45 (3), 655-689.

(3) Sutherland, B. R.; Sargent, E. H. Perovskite Photonic Sources. 307 Nat. Photonics 2016, 10 (5), 295-302.

(4) Kim, Y.-H.; Cho, H.; Lee, T.-W. Metal Halide Perovskite Light 309 Emitters. Proc. Natl. Acad. Sci. U. S. A. 2016, 113 (42), 11694-11702. 310 (5) Stranks, S. D.; Stranks, S. D.; Eperon, G. E.; Grancini, G.; 311 Menelaou, C.; Alcocer, M. J. P.; Leijtens, T.; Herz, L. M.; Petrozza, A.; 312 Snaith, H. J.; et al. Electron-Hole Diffusion Lengths Exceeding 1313 Micrometer in an Organometal Trihalide Perovskite Absorber. Science 314 2013, 342 (6156), 341-344.

(6) De Wolf, S.; Holovsky, J.; Moon, S.-J.; Löper, P.; Niesen, B.; 316 Ledinsky, M.; Haug, F.; Yum, J.; Ballif, C. Organometallic Halide 317 Perovskites: Sharp Optical Absorption Edge and Its Relation to 318 Photovoltaic Performance. J. Phys. Chem. Lett. 2014, 5, 1035-1039. 319

(7) Petrus, M. L.; Schlipf, J.; Li, C.; Gujar, T. P.; Giesbrecht, N.; 320 Müller-Buschbaum, P.; Thelakkat, M.; Bein, T.; Hüttner, S.; Docampo, 321 P. Capturing the Sun: A Review of the Challenges and Perspectives of 322 Perovskite Solar Cells. Adv. Energy Mater. 2017, 7 (16), 1-27. 323

(8) Manser, J. S.; Saidaminov, M. I.; Christians, J. A.; Bakr, O. M.; 324 Kamat, P. V. Making and Breaking of Lead Halide Perovskites. Acc. 325 Chem. Res. 2016, 49 (2), 330-338.

(9) Leijtens, T.; Bush, K.; Cheacharoen, R.; Beal, R.; Bowring, A.; 327 McGehee, M. D. Towards Enabling Stable Lead Halide Perovskite 328 Solar Cells; Interplay between Structural, Environmental, and Thermal 329 Stability. J. Mater. Chem. A 2017, 5 (23), 11483-11500.

(10) Rehman, W.; McMeekin, D. P.; Patel, J. B.; Milot, R. L.; 33 Johnston, M. B.; Snaith, H. J.; Herz, L. M. Photovoltaic Mixed-Cation 332 Lead Mixed-Halide Perovskites: Links between Crystallinity, Photo- 333 Stability and Electronic Properties. Energy Environ. Sci. 2017, 10 (1), 334 361-369.

335

(11) Kubicki, D. J.; Prochowicz, D.; Hofstetter, A.; Zakeeruddin, S. 336 M.; Grätzel, M.; Emsley, L. Phase Segregation in Cs-, Rb- and K- 337 Doped Mixed-Cation (MA)x(FA)1-xPbI3Hybrid Perovskites from 338 Solid-State NMR. J. Am. Chem. Soc. 2017, 139 (40), 14173-14180. 339

(12) Zhang, M.; Yun, J. S.; Ma, Q.; Zheng, J.; Lau, C. F. J.; Deng, X.; 340 Kim, J.; Kim, D.; Seidel, J.; Green, M. A.; et al. High-Efficiency 341 Rubidium-Incorporated Perovskite Solar Cells by Gas Quenching. 342 ACS Energy Lett. 2017, 2 (2), 438-444.

343

(13) Forgács, D.; Pérez-del-Rey, D.; Ávila, J.; Momblona, C.; Gil- 344 Escrig, L.; Dänekamp, B.; Sessolo, M.; Bolink, H. J. Efficient Wide 345 Band Gap Double Cation - Double Halide Perovskite Solar Cells. J. 346 Mater. Chem. A 2017, 5 (7), 3203-3207.

(14) Grancini, G.; Roldán-Carmona, C.; Zimmermann, I.; Mosconi, 348 E.; Lee, X.; Martineau, D.; Narbey, S.; Oswald, F.; De Angelis, F.; 349 Graetzel, M.; et al. One-Year Stable Perovskite Solar Cells by 2D/3D 350 Interface Engineering. Nat. Commun. 2017, 8, 15684.

(15) Tsai, H.; Nie, W.; Blancon, J. C.; Stoumpos, C. C.; Asadpour, 352 R.; Harutyunyan, B.; Neukirch, A. J.; Verduzco, R.; Crochet, J. J.; 353 Tretiak, S.; et al. High-Efficiency Two-Dimensional Ruddlesden- 354 Popper Perovskite Solar Cells. Nature 2016, 536 (7616), 312-317. 355

(16) Smith, I. C.; Hoke, E. T.; Solis-Ibarra, D.; McGehee, M. D.; 356 Karunadasa, H. I. A Layered Hybrid Perovskite Solar-Cell Absorber 357 with Enhanced Moisture Stability. Angew. Chem., Int. Ed. 2014, 53358 (42), 11232-11235.

(17) Cao, D. H.; Stoumpos, C. C.; Farha, O. K.; Hupp, J. T.; 360 Kanatzidis, M. G. 2D Homologous Perovskites as Light-Absorbing 361 Materials for Solar Cell Applications. J. Am. Chem. Soc. 2015, 137 (24), 362 7843-7850.

(18) Lira-Cantú, M. Perovskite Solar Cells: Stability Lies at 364 Interfaces. Nat. Energy 2017, 2, 17115. 
366 (19) Saliba, M.; Matsui, T.; Domanski, K.; Seo, J.-Y.; Ummadisingu, 367 A.; Zakeeruddin, S. M.; Correa-Baena, J.-P.; Tress, W. R.; Abate, A.; 368 Hagfeldt, A.; et al. Incorporation of Rubidium Cations into Perovskite 369 Solar Cells Improves Photovoltaic Performance. Science (Washington, 370 DC, U. S.) 2016, 354, 206.

371 (20) Wang, Q.; Chen, B.; Liu, Y.; Deng, Y.; Bai, Y.; Dong, Q.; Huang, $372 \mathrm{~J}$. Scaling Behavior of Moisture-Induced Grain Degradation in 373 Polycrystalline Hybrid Perovskite Thin Films. Energy Environ. Sci. 374 2017, 10 (2), 516-522.

375 (21) Yang, S.; Wang, Y.; Liu, P.; Cheng, Y.-B.; Zhao, H. J.; Yang, H. 376 G. Functionalization of Perovskite Thin Films with Moisture-Tolerant 377 Molecules. Nat. Energy 2016, 1 (2), 15016.

378 (22) Wang, F.; Geng, W.; Zhou, Y.; Fang, H. H.; Tong, C. J.; Loi, M. 379 A.; Liu, L. M.; Zhao, N. Phenylalkylamine Passivation of Organolead 380 Halide Perovskites Enabling High-Efficiency and Air-Stable Photo381 voltaic Cells. Adv. Mater. 2016, 28 (45), 9986-9992.

382 (23) Dequilettes, D. W.; Koch, S.; Burke, S.; Paranji, R. K.; 383 Shropshire, A. J.; Ziffer, M. E.; Ginger, D. S. Photoluminescence 384 Lifetimes Exceeding $8 \mathrm{Ms}$ and Quantum Yields Exceeding 30\% in 385 Hybrid Perovskite Thin Films by Ligand Passivation. ACS Energy Lett. 386 2016, 1 (2), 438-444.

387 (24) Huang, G.; Wang, C.; Zhang, H.; Xu, S.; Xu, Q.; Cui, Y. Post388 Healing of Defects: An Alternative Way for Passivation of Carbon389 Based Mesoscopic Perovskite Solar Cells via Hydrophobic Ligand 390 Coordination. J. Mater. Chem. A 2018, 6 (6), 2449-2455.

391 (25) Momblona, C.; Gil-Escrig, L.; Bandiello, E.; Hutter, E. M.; 392 Sessolo, M.; Lederer, K.; Blochwitz-Nimoth, J.; Bolink, H. J. Efficient 393 Vacuum Deposited P-I-N and N-I-P Perovskite Solar Cells Employing 394 Doped Charge Transport Layers. Energy Environ. Sci. 2016, 9 (11), 395 3456-3463.

396 (26) Shao, F.; Xu, L.; Tian, Z.; Xie, Y.; Wang, Y.; Sheng, P.; Wang, 397 D.; Huang, F. A Modified Two-Step Sequential Deposition Method 398 for Preparing Perovskite $\mathrm{CH}_{3} \mathrm{NH}_{3} \mathrm{PbI}_{3}$ Solar Cells. RSC Adv. 2016, 3996 (48), 42377-42381.

400 (27) Lee, W. H.; Chen, C. Y.; Li, C. S.; Hsiao, S. Y.; Tsai, W. L.; 401 Huang, M. J.; Cheng, C. H.; Wu, C. I.; Lin, H. W. Boosting Thin-Film 402 Perovskite Solar Cell Efficiency through Vacuum-Deposited Sub403 Nanometer Small-Molecule Electron Interfacial Layers. Nano Energy 404 2017, 38, 66-71.

405 (28) Tanuma, S.; Powell, C. J.; Penn, D. R. Calculations of Electron 406 Inelastic Mean Free Paths. V. Data for 14 Organic Compounds over 407 the 50-2000 eV Range. Surf. Interface Anal. 1994, 21 (3), 165-176. 